\title{
Adherencia al Protocolo de Manejo del Accidente Biológico en una Administradora de Riesgos Laborales de Colombia, 2012-2013
}

\section{ADHERENCE MANAGEMENT PROTOCOL OF OCCUPATIONAL EXPOSURE TO BLOODBORNE PATHOGEN IN AN OCCUPATIONAL RISK ADMINISTRATOR OF COLOMBIA, 2012-2013}

\author{
Machado-Alba Jorge E1, Cardona-Martínez Beatriz E. ${ }^{2}$, González-Arias Rosalba L ${ }^{3}$ \\ 1. Máster en Farmacoepidemiología, Máster en Farmacología. Profesor titular de Farmacología y Toxicología, Departamento de Ciencias Básicas, \\ Facultad de Ciencias de la Salud y Director del Grupo de Investigación en Farmacoepidemiología y Farmacovigilancia, Universidad Tecnológica de \\ Pereira-Audifarma S.A. Profesor del Posgrado en Epidemiología de la Fundación Universitaria del Área Andina de Pereira. Pereira, Colombia. \\ 2. Médica Especialista en Epidemiología, Fundación Universitaria del Área Andina de Pereira; Médica Auditora Regional Eje Cafetero, Administradora \\ de Riesgos Laborales Seguros de Vida Colpatria S.A. \\ 3. Enfermera Especialista en Epidemiología, Fundación Universitaria del Área Andina de Pereira; Coordinadora Epidemiología Secretaría de Salud \\ Municipal de Pereira.
}

\begin{abstract}
RESUMEN
Introducción: la exposición accidental ocupacional a sangre y otros fluidos corporales es uno de los más frecuentes riesgos laborales, presentándose en el personal sanitario mundial alrededor de 3 millones de pinchazos/año con objetos cortopunzantes contaminados; su adecuada atención es indispensable para prevenir enfermedades infecciosas. Objetivo: Determinar la adherencia al protocolo de manejo de los accidentes de trabajo con riesgo biológico, las variables asociadas en las atenciones iniciales, y la oportunidad en los seguimientos recomendados al trabajador en una aseguradora de riesgos laborales (ARL) de Colombia. Materiales y métodos: Se realiza un estudio descriptivo de corte transversal analizando todos los casos reportados a la ARL Colpatria entre el 1 de febrero del 2012 y el 28 de febrero de 2013. La recolección de los datos fue realizada por una entidad especializada en asesoramiento y acompañamiento de los casos mediante seguimiento telefónico, considerando variables sociodemográficas (edad, sexo, lugar de ocurrencia), propias del evento (tipo de accidente, fluido involucrado, parte de cuerpo afectada); se determinó la adherencia a la guía de manejo de acuerdo al cumplimiento de la misma. Se aplicaron pruebas de $\mathrm{X}^{2}$ y regresión logística. Resultados: se caracterizaron 1485 accidentes con riesgo biológico; los cargos como técnico en enfermería y personal encargado de la disposición de residuos presentaron la frecuencias más altas de exposición, con una proporción de 40,7 y 19,2\%, respectivamente. La adherencia al esquema de hepatitis B fue del 70\%, al de hepatitis C fue del 83\%, al de VIH de 86\% y el inicio de terapia postexposición del 89\%; la oportunidad en los seguimientos varió entre el 18 y 41. Discusión: se encuentran grandes falencias tanto en el proceso de disposición de cortopunzantes y cobertura de vacunación (prevención primaria), como en el diagnóstico de riesgo y en la oportunidad en el seguimiento de los casos estudiados (prevención secundaria).
\end{abstract}

(Machado-Alba J, Cardona-Martínez B, González-Arias R, 2014. Adherencia al Protocolo de Manejo del Accidente Biológico en una Administradora de Riesgos Laborales de Colombia, 2012-2013. Cienc Trab. May-Ago; 16 [50]: 103-110).

Palabras claves: EXPOSICIÓN OCUPACIONAL, FLUIDO CORPORAL, GUÍAS DE PRÁCTICA CLÍNICA, ADHERENCIA.

\section{ABSTRACT}

Introduction: accidental occupational exposure to blood and other body fluids, is one of the most common occupational risks. In the world suffers medical staff about 3 million needlestick / sharps contaminated year, adequate care is essential to prevent and / or early detection of infectious diseases. Objective: To determine adherence to protocol management of occupational accidents with biological risk associated variables in the initial care, and recommended follow-ups opportunity to work in a labor risk insurance (ARL) of Colombia, Materials and methods: We performed a cross-sectional descriptive study analyzing all cases reported to the ARL Colpatria between February 1, 2012 and February 28, 2013. The data collection was carried out by an entity specializing in advice and support by telephone follow-up cases, considering sociodemographic variables (age, sex, place of occurrence), own the event (type of accident involved fluid, body part affected) was determined adherence to management guidelines according to the fulfillment of the same. $\mathrm{X}^{2}$ tests were applied and logistic regression. Results: Were characterized 1485 cases of occupational exposure to bloodborne pathogen, the technician charges in nursing and staff responsible for waste disposal had the highest frequency exposure with a ratio of 40.7 and $19.2 \%$ respectively. Scheme adherence hepatitis B was 70\%, that of hepatitis C was $83 \%$, the HIV of $86 \%$ and post-exposure therapy was started 89\%, the opportunity in traces ranged between 18 and 41 . Discussion: are major flaws in the process both sharps disposal and vaccination coverage (primary prevention) and in the diagnosis of risk and the opportunity to monitor the cases studied (secondary prevention).

Key words: OCCUPATIONAL EXPOSURE, BODY FLUID, CLINICAL PRACTICE GUIDELINES, ADHERENCE.
Correspondencia / Correspondence:

Beatriz Elena Cardona Martínez

Calle $37 \mathrm{~N}^{\circ} 13-108$, Conjunto residencial Niza I, Bloque 6 Apto 4B

Pereira, Risaralda, Colombia, código postal: 660004

Tel.: (57-6)-3266310 / móvil: 57- 311-6340082

e-mail: beatriz.cardona@axacolpatria.com; bettycam76@hotmail.com.

Recibido: 31 de Mayo de 2014 / Aceptado: 15 de Junio de 2014

\section{INTRODUCCIÓN}

En el mundo laboral existen diferentes niveles de riesgo para la salud de los trabajadores directamente relacionados con las actividades cotidianas; específicamente, en el caso de accidente de trabajo con riesgo biológico, los trabajadores que llevan a cabo actividades laborales relacionadas con las ciencias de la salud y/o los recolectores de desechos presentan una exposición continua al 
contagio de enfermedades virales por transmisión sanguínea, tales como: Virus de inmunodeficiencia humana (VIH), Virus de la hepatitis B (VHB) y virus de la hepatitis C (VHC), presentando cada una un comportamiento epidemiológico diferente $\mathrm{y}$, por tanto, un riesgo independiente para la población trabajadora involucrada. El advenimiento de la pandemia del VIH, desde la publicación de su primer caso en 1981, ha tenido un abrumador crecimiento a nivel mundial, registrándose casos en cada uno de los países del mundo con cifras de contagio de la OMS estimadas para el año 2011 de hasta 34.2 millones de personas infectadas ${ }^{1,2}$; también este organismo ha alertado sobre el incremento de los accidentes con riesgo biológico en el mundo. Según estadísticas de esta organización, se estima que el personal sanitario sufre alrededor de 3 millones de pinchazos/año con objetos cortopunzantes contaminados (lancetas y agujas, vidrios rotos o bisturí), presentándose aproximadamente entre 300.000 y 800.000 casos/año en Estados Unidos, aunque la distribución en la práctica ambulatoria u hospitalaria es desconocida. $^{3-6}$ Según el sistema de información en salud ocupacional implementado en varios países del mundo, EPINET, se estima una tasa de incidencia, en España, de 11,8 casos por 100 camas (similar a otros países de Europa); y, en Estados Unidos, Canadá y Australia tasas de 20 a 33\% de casos. ${ }^{7}$ En la actualidad no se dispone de datos periódicos sobre el número de eventos ocurridos en Colombia, ya que no se cuenta con un sistema de información similar o de vigilancia del evento que permita cuantificar y caracterizar este tipo de accidentes laborales. Aunque los trabajadores del sector salud son los más frecuentemente afectados con este tipo de exposiciones, existe otro tipo de actividades económicas y/o sociales que también presentan factores de riesgos por exposición ocupacional biológica, como son los trabajadores de recolección de basuras, personal de servicios generales, trabajadores en áreas de esterilización, tanatólogos, personas con trabajos de rescate, personal de seguridad, entre otros. ${ }^{3,4}$ Este incremento en el número de casos se estima ha sido influenciado por factores como aumento en: el número de trabajadores sanitarios expuestos, el número de personas infectadas con virus potencialmente transmisores relacionados con la implementación de nuevas terapéuticas, el número de procedimientos invasivos derivados del advenimiento de nuevas tecnologías en salud y la capacidad de invasividad individual de cada virus. ${ }^{4}$ En el mundo se calcula que para el 2006, había aproximadamente $39^{\prime} 470.000$ trabajadores sanitarios, correspondiendo 12'460.000 de ellos a la región de las Américas. ${ }^{6,8}$ En Colombia, la población trabajadora afiliada al sistema general de riesgos profesionales (Administradoras de Riesgos Laborales, ARL), para el 2011 era de 7'484.756 trabajadores, con una tasa general de accidentalidad del 7,3\%/año. La población afiliada del sector salud, para el mismo año, representa el 5,4\% de los trabajadores, con una tasa de accidentalidad calculada del 7,1.9

Se ha definido Accidente Biológico por la OMS como: "la exposición de piel no intacta (ya sea por una lesión con objeto punzante o abrasión) o una membrana mucosa, a un fluido corporal potencialmente infectado desde una fuente humana positiva o en estado desconocido", ${ }^{3,4}$ Con este tipo de exposiciones, se presenta el riesgo de contagio de enfermedades virales por transmisión sanguínea tales como: Virus de inmunodeficiencia humana (VIH), y hepatitis virales tipo B y C, cada una de las cuales presenta un comportamiento epidemiológico diferente $\mathrm{y}$, por tanto, un riesgo independiente para la población trabajadora involucrada. Según estudios previamente realizados para $\mathrm{VIH}$, el riesgo de infección alcanza el $0.3 \%$, en el caso de la exposición percutánea, mientras que el riesgo de infección tras salpicaduras de fluidos potencialmente infectantes a membranas mucosas es del 0,09\%. En el caso de la hepatitis C, este riesgo es aproximadamente del 1.8\% (rango del 0-7\%) en exposición percutánea, desconociéndose la cifra respecto a la exposición a mucosas. ${ }^{11-13}$ Finalmente, la hepatitis $\mathrm{B}$, que es considerada como una enfermedad ocupacional, con una prevalencia 10 veces mayor en los trabajadores de la salud respecto a la población general ${ }^{14}$, la tasa de infección postexposición para el personal no inmunizado oscila, según el estado serológico de la fuente, entre el 1 al 6\% para desarrollar hepatitis clínica y el 23-37\% para que se presente evidencia serológica de la infección cuando es: Antígeno de Superficie para Virus de la Hepatitis B positivo $(\mathrm{Ag} \mathrm{HBs}+$ ) con Antígeno E para el Virus de la hepatitis B negativo (Ag $\mathrm{HBe}-$ ). Mientras que en el caso de que la fuente tenga ambos positivos, el riesgo de desarrollar hepatitis clínica es del 22 al 31\% y, de presentar evidencia serológica, del 37 al 62\%. La transmisión percutánea, aunque efectiva para el virus de la hepatitis $\mathrm{B}$, no es la más frecuente; muchos trabajadores no recuerdan haber tenido lesiones percutáneas, aunque algunos recordaron haber cuidado pacientes con $\mathrm{Ag} \mathrm{HBs}+.12,14 \mathrm{La}$ terapia postexposición para VIH (terapia PEP VIH) disminuye la probabilidad de infección en un $81 \%$ 8,14, mientras que la terapia PEP para hepatitis $B$ con esquema de vacunación y aplicación de Globulina inmune para hepatitis B la puede disminuir hasta en un $85 \% .^{13,15,16}$

El uso de guías que mejoren la calidad en la atención y el pronóstico de los pacientes debe ser un objetivo de los médicos y del personal de salud de hoy. ${ }^{17,18}$ Aunque respecto a los accidentes con riesgo biológico no hay publicaciones que verifiquen la adherencia a alguna guía establecida para su manejo, se presume un déficit en el cumplimiento de protocolos de atención en Latinoamérica y Colombia; no se encontraron datos adicionales que tengan en cuenta el nivel de adherencia en las atenciones de urgencias al protocolo de manejo en el abordaje inicial y en la oportunidad en los seguimientos realizados a los trabajadores expuestos. La adherencia, en ambos casos, es pilar fundamental del éxito en la prevención secundaria para este tipo de pacientes, por lo que se hace necesario evaluarla. En este estudio se pretende realizar un acercamiento a esta problemática y determinar la adherencia al protocolo de manejo de los accidentes de trabajo con riesgo biológico en las atenciones iniciales de los servicios de urgencias de Colombia, basados en las guías del "Center for Diseases control and Prevention (CDC)" y la oportunidad en los seguimientos recomendados al trabajador, en una ARL desde el 1 febrero del 2012 al 28 de febrero del 2013.

\section{MATERIALES Y MÉTODOS}

Población: Se realiza un estudio de tipo descriptivo de corte transversal, a partir de una fuente de información secundaria, de la totalidad de los casos reportados a una ARL de Colombia, evaluando la adherencia en el abordaje inicial con la información de los casos reportados entre el 1 de febrero del 2012 y el 28 de febrero de 2013 (13 meses) (n:1574) y seleccionando para el análisis de la oportunidad en el seguimiento, solo aquellos casos ocurridos entre el 1 de febrero y el 30 de julio de $2012(n=656)$, con el fin de garantizar que contaran con toda la información del seguimiento hasta los 6 meses sugeridos por las guías del CDC/ MMWR 20016, las cuales son el referente de manejo de este tipo 
de eventos en el mundo. La recolección de los datos fue realizada por una IPS especializada en asesoramiento y acompañamiento a los casos de ATRB, denominada AVENIR salud, la cual realiza la atención posterior al abordaje inicial en urgencias y el seguimiento a los diferentes eventos y conductas de intervención ya sean diagnósticas o terapéuticas, posteriores a la atención inicial, de todos los ATRB que les sean reportados, en las áreas de influencia de esta ARL en casi todo el país, a excepción de los departamentos de Santander y Norte de Santander, los cuales cuentan con otra entidad de apoyo. Debido al proceso de gestión, tan articulado entre la línea de asistencia de autorizaciones de la ARL y esta entidad, se puede garantizar casi la totalidad de eventos de este tipo realmente reportados, a excepción de los eventos direccionados por Empresas Promotoras de Salud (EPS), los cuales son mínimos. Los datos respecto a las conductas en los diferentes servicios de urgencias se obtuvieron utilizando un método de contrastación, consistente en que las acciones tomadas por la empresa Avenir son las complementarias para cada caso; así por ejemplo, si un evento tiene registrado en la base de datos, del abordaje inicial un servicio de laboratorio para AgHbs de la fuente o del trabajador, se concluye que no se realizó dicha prueba en la atención inicial de urgencias.

Una vez obtenida la información se realizó un proceso de verificación, depuración y concatenación de la información de las dos bases de datos originalmente suministradas, con el fin de encontrar registros contradictorios y corregirlos, apoyados en el acceso al software de información de la ARL. Se seleccionaron las siguientes variables para el análisis:

Socio-demográficas: Edad, sexo, ocupación, departamento y tipo de ciudad de ocurrencia del evento.

Características clínicas del evento: tipo de accidente, tipo de fuente de contagio, parte del cuerpo afectada, agente o instrumento que ocasiona la lesión, fluido involucrado, momento de uso del agente cortopunzante, propósito de la actividad realizada al momento del evento, antecedente de aplicación de vacuna para HB en el trabajador y accidentes previos.

Abordaje Paraclínico y seguimiento: se definieron cuatro variables de adherencia, uno de oportunidad en el reporte, y tres de oportunidad en el seguimiento, fundamentados en las recomendaciones descritas en la guías del CDC/MMWR 2001- 2005. ${ }^{12,13}$

Los criterios de adherencia y oportunidad son los siguientes:

Adherencia al esquema de hepatitis B: Se evalúa la realización del AgHBs (antigeno de superficie del virus de la hepatitis B) en la fuente conocida y el trabajador. Y la prescripción de Ac HBs (anticuerpo para AgHBs) únicamente al trabajador.

Adherencia al esquema de laboratorio VIH: Se evalúa la prescripción y/o realización del examen de la prueba ELISA para VIH al trabajador y a la fuente conocida.

Adherencia al esquema de la prescripción de la terapia PEP VIH: Se evaluaron cambios en la terapéutica prescrita, como el inicio o suspensión del tratamiento profiláctico.

Adherencia al esquema de Hepatitis C: Se evalúa la prescripción de Ac HC (Anticuerpos para Hepatitis C) al trabajador y/o a la fuente según corresponda.

Adicionalmente se valoró la oportunidad en el reporte a la empresa, teniendo en cuenta la fecha de ocurrencia del accidente, considerándose como oportuno cuando se reportó en las primeras 24 horas. Definición de criterios de oportunidad para los seguimientos serológicos: Primer seguimiento: la recomendación es realizarlo de las 4 a 6 semanas posterior al evento. ${ }^{12,13}$ Por lo que a criterio de los investigadores se definió como oportuno los realizados entre los 28 y 45 días posteriores al mismo. Segundo seguimiento: La recomendación es realizarlo a las 12 semanas posteriores al evento. Por lo que, a criterio de los investigadores, se definió como oportuno un rango de tiempo entre 80 y 99 dias. Tercer seguimiento: La recomendación es realizarlo a los 6 meses posteriores al evento. Por lo que se definió como oportuno un rango de tiempo entre 180 y 210 días. Los resultados se obtuvieron por medio análisis univariado con EPIDAT4.0. Los valores calculados y las mediciones se presentan como media \pm desviación estándar para las variables continuas y como porcentaje para las variables discretas. El análisis bivariado y multivariado se hizo por medio del paquete estadístico SPSS 19.0 para Windows. En Las variables categóricas se utilizó $\mathrm{X}^{2}$ (chi 2) como medida de asociación. En el análisis multivariado, se tomó como variable dependiente la adherencia a los diferentes esquemas y las independientes todas aquellas que estén asociadas de manera significativa en el análisis bivariado con análisis de regresión logística. El proceso se realiza previa autorización de la gerente de dicha entidad y del Director nacional de ARL y Salud Colpatria además fue avalado por el comité de bioética de la Universidad Tecnológica de Pereira y representa un riesgo mínimo para los participantes de acuerdo a la resolución 8430 de 1993 del Ministerio de Salud de Colombia, garantizando los principios de beneficencia, no maleficencia $\mathrm{y}$ confidencialidad.

Limitantes: Este estudio solo evalúa los casos reportados como accidente de riesgo biológico a la ARL Colpatria, por lo que los resultados dependen directamente de la composición poblacional afiliada a esta institución; por tanto, en cuanto a datos demográficos, no se podría extrapolar estos resultados al resto de la población. Asimismo, no fue posible evaluar la conducta de los trabajadores en relación al cumplimiento en la terapia PEP para VIH.

\section{RESULTADOS}

De los 1574 eventos contenidos en la base de datos consolidada, se excluyó un total de 89 casos (5,6\%); en 21 de ellos (1,3\%) no se pudo establecer contacto con el trabajador; 1 más, por razones administrativas; 6 porque el mecanismo de exposición al riesgo biológico, no hacían parte del objeto de este estudio (2 por abuso sexual, 4 por exposición a meningitis); y 61 que, en concepto de los investigadores, no cumplían con la definición para exposición a riesgo biológico de transmisión sanguínea. De tal forma que finalmente se realizó el análisis de adherencia en un total de 1485 eventos. La edad promedio fue de 31,9 \pm 8 ,9 años, con una distribución por sexo de 1180 mujeres (79,5\%) y 305 hombres (20,5\%). La experiencia laboral en promedio fue de 6,2 \pm 6,4 años, donde un 13,2\% de los afectados tienen experiencia laboral menor a un año. Los cargos como técnico en enfermería, 40,7\% (605 casos), y personal encargado de la disposición de residuos, 19,2\% (285), presentó las frecuencias más altas de ATRB (ver Gráfico 1).

El número de casos ocurridos en las ciudades principales corresponde al 83.0\% (1232), sin ningún reporte en los departamentos de Chocó, Guaviare, Guainía, Putumayo, Meta, Vaupés y Vichada (Gráfico 2).

Se observó que el lugar de más frecuente ocurrencia fue el hospitalario, con un 84\%, (hospitalización con 23,1\%, urgencias 19,7\% y procedimientos 11,7\%); el tipo de accidente que ocupó la mayor 


\section{Gráfico 1.}

Número de casos según ocupación y esquema HB.

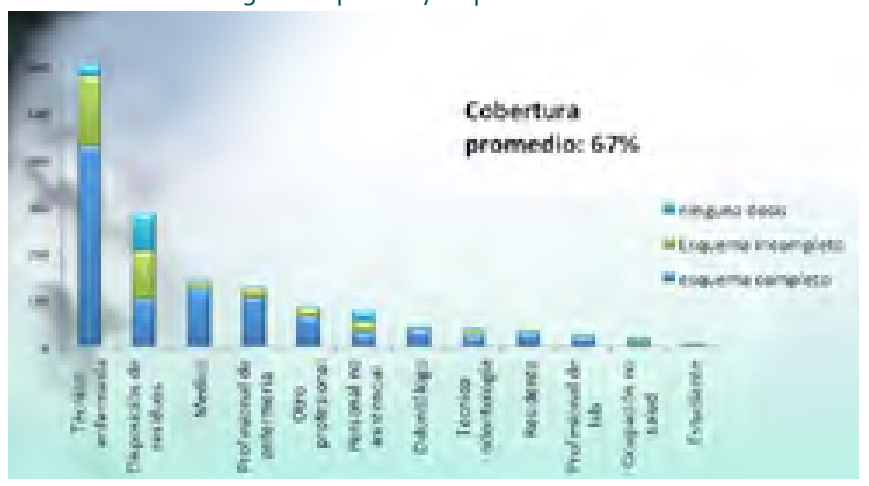

Gráfico 2.

Distribución de los eventos en el país.

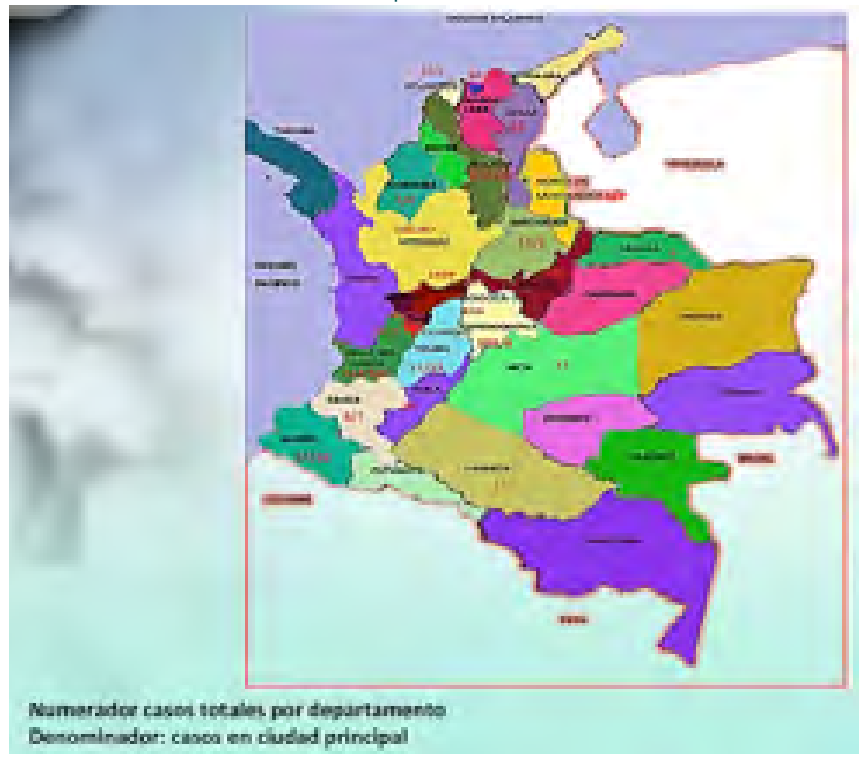

Tabla 1.

Caracterización de los accidentes por vía percutánea y mucosa, según los factores asociados que aumentan el riesgo.

\begin{tabular}{|c|c|c|c|c|c|}
\hline $\begin{array}{l}\text { Factor de riesgo } \\
\text { asociado con severidad }\end{array}$ & Tipo de exposición & \multicolumn{2}{|c|}{$\begin{array}{c}\text { Percutáneos } \\
\mathrm{N}=1337(90 \%)\end{array}$} & \multicolumn{2}{|c|}{$\begin{array}{c}\text { Mucosos } \\
\mathrm{N}=148(10 \%)\end{array}$} \\
\hline \multirow{7}{*}{$\begin{array}{l}\text { Tipo de agente } \\
\text { involucrado }\end{array}$} & Variable descrita & $\mathrm{N}^{\circ}$ & $\%$ & $\mathrm{~N}^{0}$ & $\%$ \\
\hline & Aguja hueca & 946 & $70,8 \%$ & & \\
\hline & Punzante no hueco & 260 & $19,4 \%$ & & \\
\hline & Instrumental & 58 & $4,3 \%$ & & \\
\hline & Material cortante & 54 & $4,0 \%$ & & \\
\hline & Desconocido & 18 & $1,3 \%$ & & \\
\hline & Dientes, uñas & 1 & $0,1 \%$ & & \\
\hline \multirow{3}{*}{$\begin{array}{l}\text { Fluido corporal } \\
\text { involucrado }\end{array}$} & Sangre & 957 & $71,6 \%$ & 99 & $66,9 \%$ \\
\hline & Fluido desconocido & 343 & $25,7 \%$ & 42 & $28,4 \%$ \\
\hline & Otros fluidos & 37 & $2,8 \%$ & 7 & $4,7 \%$ \\
\hline \multirow{7}{*}{$\begin{array}{l}\text { Propósito del } \\
\text { procedimiento } \\
\text { relacionado con } \\
\text { contacto con } \\
\text { sangre } \\
\mathrm{N}=\text { (957) }\end{array}$} & No contact & 434 & $45,4 \%$ & & \\
\hline & Contacto con sangre venosa & 232 & $24,2 \%$ & & \\
\hline & Contacto con sangre arterial & 121 & $12,6 \%$ & & \\
\hline & Otro tipo de muestra & 121 & $12,6 \%$ & & \\
\hline & Procedimientos anestesia oral & 11 & $1,1 \%$ & & \\
\hline & Desconocidos & 17 & $1,8 \%$ & & \\
\hline & $\begin{array}{l}\text { No participa en el } \\
\text { proceso de desecho }\end{array}$ & 21 & $2,2 \%$ & & \\
\hline \multirow{3}{*}{$\begin{array}{l}\text { Estado de vacunación } \\
\text { referido por } \\
\text { el paciente }\end{array}$} & Completo & 888 & $66,4 \%$ & 107 & $72,3 \%$ \\
\hline & Incompleto & 307 & $23,0 \%$ & 31 & $20,9 \%$ \\
\hline & Ninguno & 142 & $10,6 \%$ & 10 & $6,3 \%$ \\
\hline
\end{tabular}

**No hay datos disponibles sobre sangre visible en el dispositivo o profundidad de la lesión medidos de forma objetiva \& únicamente los relacionados con sangre.
Tabla 2.

Adherencia a los esquemas diagnósticos y terapéuticos evaluados, en el abordaje inicial de los eventos con exposición ocupacional a patógenos de transmisión sanguínea.

\begin{tabular}{lcccc} 
Variable de & $\begin{array}{c}\text { Número de } \\
\text { Adherentes }\end{array}$ & \multicolumn{3}{c}{ Número de } \\
Adherencia & 1316 & $89 \%$ & 169 & $11 \%$ \\
\hline Adh. Esquema PEP VIH $\$$ No Adherentes & $\%$ & 207 & $14 \%$ \\
Adh. Esquema exámenes VIH & 1278 & $86 \%$ & 247 & $17 \%$ \\
Adh. Esquema hepatitis C & 1238 & $83 \%$ & 443 & $30 \%$ \\
Adh. Esquema Hepatitis B & 1042 & $70 \%$ & 563 & $37,9 \%$
\end{tabular}

\$ se considera no adherente cuando se registra "se inicia tratamiento profiláctico" o "se suspende tratamiento profiláctico iniciado en atención inicial". En caso contrario se considera como adherente.

\& se evalúa la prescripción y/o realización del examen de la prueba ELISA para VIH al trabajador y/o la fuente conocida según sea el caso.

I se evalúa la prescripción de Ac HC (Anticuerpos para Hepatitis A11) al trabajador y/o a la fuente según corresponda.

* se evalúa la conducta respecto a la realización del Ag HBs (antígeno de superficie del virus de la hepatitis B) en la fuente conocida y el trabajador y Ac HBs (anticuerpo para $\mathrm{Ag} \mathrm{HBs)} \mathrm{al} \mathrm{trabajador} \mathrm{únicamente.}$

$\Omega$ que presentan adherencia a esquemas evaluados.

\section{Gráfico 3.}

Momento de ocurrencia de la exposición, relacionado con el uso del agente cortopunzante.

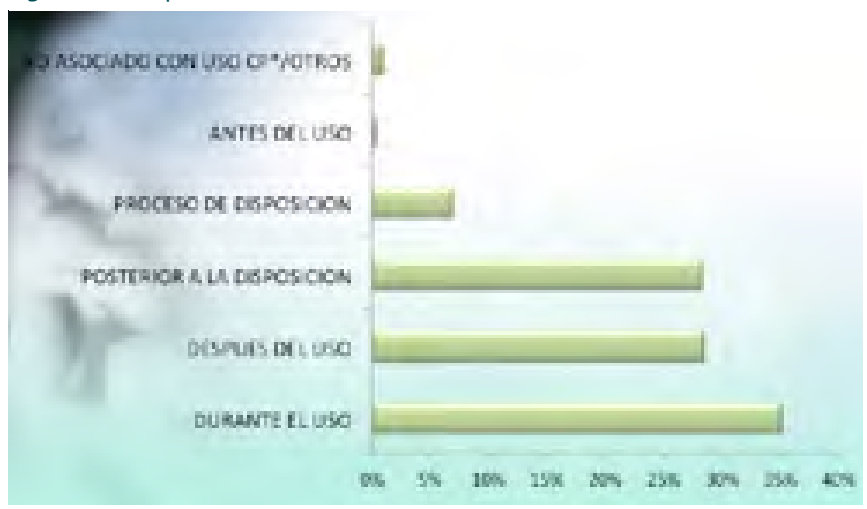

proporción fue el percutáneo (90,0\%), seguido de 8,15\% con exposición a mucosas, el valor restante correspondió a contacto con piel no intacta; siendo las manos (con el 91,0\%), la parte del cuerpo que más se vio afectada para los percutáneos y los ojos para la exposición a mucosas. La fuente fue conocida en el 58,38\% (867) de todos los casos. Las características de los accidentes percutáneos y mucosos, asociados con el riesgo se relacionan en la Tabla 1 y 2 respectivamente, aunque no fue posible con la información disponible, evaluar datos como fase de diagnóstico de la fuente, sangre visible en el dispositivo o cantidad de fluido.

Respecto al momento de ocurrencia, en el caso de los accidentes percutáneos, la mayor parte ocurrieron durante el uso del objeto cortopunzante; sin embargo, el 35,2\% ocurrió en el proceso de disposición y posterior a ella (Gráfico 3).

La adherencia a cada uno de los esquemas se puede apreciar en la Tabla 3. La adherencia varió entre 70,2 y el 88,6\% de los casos, y solo el 62,0\% fue adherente a los tres esquemas.

\section{Análisis bivariado}

Al realizarse el análisis bivariado se encuentran múltiples asociaciones estadísticamente significativas de las variables dependientes con las diferentes variables independientes. La adherencia al esquema hepatitis $\mathrm{B}$ se encontró asociada estadísticamente con 
Tabla 3.

Variables asociadas a la adherencia diagnóstico de riesgo y Terapia PEP $\mathrm{VIH}$, de accidentes con riesgo biológico, en modelos de regresión logística binaria, Colombia, 2012-2013.

\begin{tabular}{|c|c|c|c|c|c|c|c|c|}
\hline Variables asociadas & B & E.E & Wald & $\mathrm{Gl}$ & Sig. & RR & $\begin{array}{l}\text { I.C. } 95 \% p \\
\text { Inferior }\end{array}$ & $\begin{array}{r}\text { axa EXP(B) } \\
\text { Superior }\end{array}$ \\
\hline \multicolumn{9}{|l|}{ Esquema Hepatitis B } \\
\hline $\begin{array}{l}\text { Antecedente } \\
\text { de accidente } \\
\text { Primera vez }\end{array}$ & 0,8739 & 0,279 & 9,815 & 1 & 0,002 & 2,396 & 1,387 & 4,14 \\
\hline $\begin{array}{l}\text { Área de trabajo } \\
\text { Ambulancia }\end{array}$ & 0,928 & 0,372 & 6,24 & 1 & 0,012 & 2,53 & 1,221 & 5,242 \\
\hline \multicolumn{9}{|l|}{ Esquema examen VHI } \\
\hline $\begin{array}{l}\text { Área de trabajo } \\
\text { Laboratorio clínico }\end{array}$ & 0,59 & 0,257 & 5,284 & 1 & 0,022 & 1,803 & 1,091 & 2,982 \\
\hline \multicolumn{9}{|l|}{ Esquema Hepatitis C } \\
\hline $\begin{array}{l}\text { Agente } \\
\text { Punzante no hueco }\end{array}$ & $-0,605$ & 0,218 & 7,71 & 1 & 0,005 & 0,546 & 0,356 & 0,837 \\
\hline $\begin{array}{l}\text { Antecedente esquema } \\
\text { de vacunación para HB } \\
\text { Esquema completo }\end{array}$ & $-0,368$ & 0,153 & 5,797 & 1 & 0,016 & 0,692 & 0,513 & 0,934 \\
\hline \multicolumn{9}{|c|}{ Esquema Terapia PEP VIH } \\
\hline $\begin{array}{l}\text { Fuente } \\
\text { Conocida }\end{array}$ & $-0,924$ & 0,248 & 13,859 & 1 & 0 & 0,397 & 0,244 & 0,646 \\
\hline $\begin{array}{l}\text { Propósito o actividad } \\
\text { Desconocido }\end{array}$ & 0,755 & 0,339 & 4,959 & 1 & 0,026 & 2,128 & 1,095 & 4,137 \\
\hline
\end{tabular}

variables como haber tenido más de un accidente $(\mathrm{p}<0,001)$ y la ocurrencia en ambiente hospitalario con un 7\% más de adherencia (71\% respecto a 64\% en los servicios restantes $(\mathrm{P}=0,027)$. Respecto al área de trabajo, los funcionarios relacionados con el transporte ambulancia $(\mathrm{p}=0,030)$ y trabajar en el laboratorio presenta menores proporciones de adherencia $(p=0,021)$. Para la variable Adherencia al esquema VIH se presentó asociación con variables como el cargo técnico en odontología ( $p=0,020)$, trabajar en un laboratorio $(p=0,021)$ o tipo de agentes como instrumental quirúrgico $(p=0,022)$ con un menor cumplimiento en el manejo y el tipo de agente punzante no hueco con una mayor adherencia $(\mathrm{p}=$ 0,027). Al igual que en los dos anteriores, en el esquema de manejo para hepatitis $\mathrm{C}$ se observó una asociación estadísticamente significativa con el cargo del trabajador con menor adherencia en los trabajadores de disposición de residuos $(\mathrm{p}<0.01)$ y técnicos en odontología ( $\mathrm{p}=0,03$ ); $\mathrm{y}$ tal como ocurrió, en el caso del esquema VIH se presentó una mayor adherencia en los casos donde el agente causal fue un objeto punzante no hueco $(p=0,03)$.

En el esquema PEP VIH, se encontró una mayor adherencia, en algunas variables sociodemográficas como el sexo, con un $94 \%$ en las mujeres y un $84,0 \%$ en los hombres ( $p=0,004$, OR: 0,59; IC
95\%: 0,4- 0,8); el cargo u ocupación médico con una adherencia del 96,0\% frente al 88,0\% en los restantes ( $p=0,013$, OR: 2,58; IC 95\%: 1,2 - 5,6), técnico en enfermería un 91,0\% de adherencia frente a un 87,0\% ( $p=0,005$, RR: 1,6;IC 95\%: 1,2-2,3); por el contrario, nuevamente en cargos como técnico en odontología $\mathrm{y}$ disposición de residuos se observó una menor adherencia. Respecto a las características propias de presentación del evento, los casos en que la fuente fue conocida se presentó una mayor adherencia: 95\% frente a un 79\% en la fuente desconocida ( $<<0,001$, RR 5,08; IC95\%: 3,5-7,3). De nuevo los ocurridos en el ambiente hospitalario presentaron mejor adherencia que los restantes lugares, con un $92 \%$ frente a un $77 \%(p<0,001)$.

$\mathrm{Al}$ analizar mediante regresión logística binaria, la relación entre la adherencia a estos esquemas y las variables que previamente presentaron asociación en el análisis bivariado se encontró, como se observa en la Tabla IV, las covariables cargo, como los trabajadores del área de ambulancias y de laboratorio clínico, presentaron un menor cumplimiento del esquema de hepatitis B y paraclínicos para VIH respectivamente, al igual que si el accidente ocurrido es por primera vez, aunque este último únicamente en el caso de la adherencia para hepatitis B. De manera similar, se presenta menor adherencia en el caso de la prescripción de la terapia PEP, si se desconoce el propósito de la actividad realizada con el agente al momento del evento. De forma complementaria se presentó una mayor adherencia cuando el agente causal fue punzante no hueco, el trabajador tenía el esquema completo para hepatitis B o la fuente era conocida.

Como se puede ver en la Tabla V, la oportunidad medida para el reporte inicial (en las primeras 24 horas) fue del 88,5\% (1315/1485). En cuanto a los seguimientos $(n=656)$, estos oscilaron entre el $19,1 \%$ y el 42,8\%, teniendo en cuenta que el 59\%, presentó registro de por lo menos un seguimiento serológico, y el 55,3\% continuaba en control, aun después de los 6 meses.

Respecto a las variables que se asociaron con la oportunidad en los seguimientos, como se observa en la Tabla VI, se encontró que los trabajadores relacionados con la disposición de residuos presentaron una menor oportunidad en el primer y segundo seguimiento, $5,1$ frente al 19-23\% en las demás ocupaciones ( $\mathrm{p}<0,001)$, mientras que los técnicos en enfermería fueron más oportunos, con un $21 \%$ frente al $13 \%$ de los cargos restantes $(p=0,01)$. Al contrario de lo que podría pensarse, los casos ocurridos en las ciudades principales presentan menor oportunidad que los de los municipios: 17,5 frente al 26,8\% ( $\mathrm{p}=0,022)$.

Adicionalmente se realizó un consolidado sobre el estado final de

Tabla 4.

Análisis bivariado de las características sociodemográficas, en 656 pacientes que presentaron accidente de trabajo con riesgo biológico, según oportunidad al esquema de seguimiento propuesto por la guía del CDC, MMRW 2001 y 2005, en una ARL de Colombia, febrero a julio del 2012.

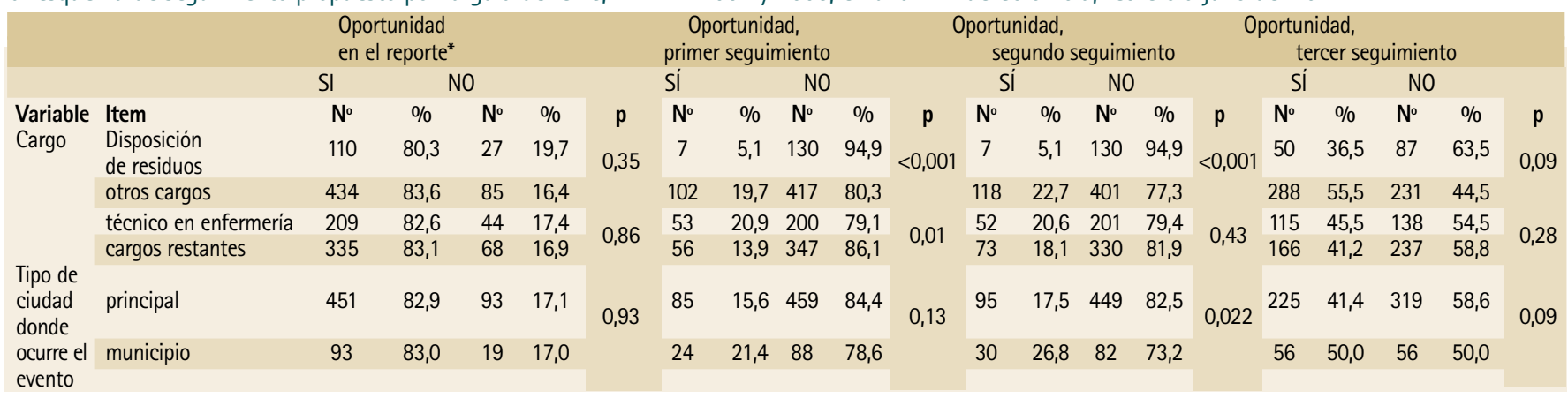


los seguimientos de los eventos, a pesar de la ya mencionada búsqueda activa de los casos (Gráfico 4).

Finalmente, de acuerdo al número de empleados de la entidad aseguradora, se calculó que ésta contó con 1'015.186 trabajadores afiliados/mes; en el transcurso de 12 meses se presentaron 1380 eventos, de los cuales, se restaron 21 eventos reportados origina-

\section{Gráfico 4.}

Estado final de los casos, inoportunos y perdidos.

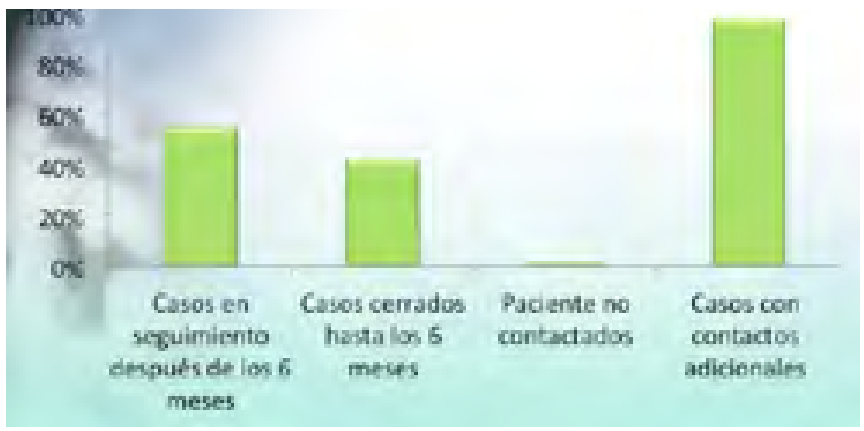

rios de los mismos departamentos, con lo cual se calculó sobre 1359 casos, para un número mensual promedio de 113 eventos, obteniéndose una tasa de prevalencia de 11 casos/mes por cada 100.000 trabajadores afiliados a la ARL, sin contar con los departamentos de Santander y Norte de Santander.

\section{DISCUSIÓN}

La tasa de incidencia de ATRB en algunas regiones es calculada según el número de camas hospitalarias, por medio del sistema de información disponible en España y otros países de Europa, EPINETAC (Exposure Prevention Information Network), y EPINET o NaSH, implementado por el CDC desde 1995 en Estados Unidos. Se estima una tasa de incidencia, en España, de 11,8 casos por 100 camas (similar a otros países de Europa), mientras que en Estados Unidos, Canadá y Australia se calculan tasas de 20 a $33 \% .^{7}$ En Colombia no hay disponibilidad de este tipo de datos. Sin embargo, en el presente estudio y ante la información disponible de la población trabajadora afiliada se calculó una tasa de prevalencia de 11 casos/mes por cada 100000 trabajadores afiliados a la ARL, sin contar con los departamentos de Santander y Norte de Santander. Es llamativo que de acuerdo con las características sociodemográficas de la población trabajadora afectada en países como España, en donde predomina el personal de salud con un 91,0\% de los afectados y menos del 5\% de ocupaciones relacionadas con servicios generales ${ }^{7}$, en este estudio se observó una proporción del $19,2 \%$ de trabajadores relacionados con servicios generales y recolección de residuos, ascendiendo al 25,7\% cuando se incluyen otros cargos como personal no asistencial (camilleros, ascensoristas); aunque se mantiene el personal de salud como el más afectado, siendo los cargos más representativos el personal técnico en enfermería y los médicos con el 40,74\% (605) y el 9,36\% (139), respectivamente.

Referente a las características propias de las exposiciones, se encontraron datos similares a los internacionales. En este estudio la proporción de accidentes por vía percutánea fue del 89,0\% ( $\mathrm{n}=$ 1337/1485), siendo similares al 88,0\% registrado en Estados Unidos3 y del 92,0\% de las exposiciones en España. ${ }^{7} \mathrm{Al}$ igual que el porcentaje de responsabilidad del agente causal con un 70,0\% de punzantes huecos ( $n=946 / 1337)$, frente a un 69\% descrito en España. ${ }^{7}$ Sin embargo, en cuanto al tipo de fuente, se observó una diferencia importante, con una proporción de accidentes con fuente conocida del 58,4\% ( $\mathrm{n}=867 / 1485)$, comparado con datos del CDC en Estados Unidos, donde esta proporción es del 91,1\% (n $=25510 / 28010)^{13}$, lo que puede aumentar en gran medida el riesgo de seroconversión en los trabajadores y de manera consecuente los costos debido al mayor número de casos a los cuales se les debe realizar seguimientos al no conocer el estado de la fuente. A lo anterior se suma que el momento de ocurrencia del evento tipo percutáneo y, aunque los datos a comparar publicados a este respecto no son totalmente equiparables -ya que se basaron sobre población trabajadora ya infectada posterior a una exposición-, se describe que el 20,0\% ocurrió durante la destrucción del agente con un 41,0\% que ocurrió después del procedimiento frente a un $7 \%$ y un $28,4 \%$, respectivamente, en el presente estudio, mientras que durante el procedimiento se describe la misma proporción en ambos del 35,0\%. ${ }^{3,18}$ Finalmente, un 35,2\% de los accidentes de tipo percutáneo ocurre durante y posterior a la disposición del agente (Gráfico 3). En el presente estudio se establece un acierto en la prescripción o no de terapia PEP del 89,0\% de los casos, presentándose cambios (suspensión o inicio de la misma), por parte de la línea de apoyo, en el 11,0\%. Sin embargo, no se obtienen datos acerca del cambio de terapia, ni tampoco datos directos sobre el estado de la fuente, por parte del servicio de apoyo, de modo que la información no puede ser comparada con los estudios descritos en CDC-MMWR/Guidelines del 2005, en donde se referencia diferentes conductas en cuanto a la prescripción, como cambios en los esquemas, adición de medicamentos o dosis, etc. A lo anterior se le suma que en este mismo estudio se tienen en cuenta únicamente los accidentes con fuente conocida, sin describir la conducta frente a los accidentes de riesgo biológico con fuente desconocida, probablemente debido a que en dicha población esta proporción equivale apenas al $8,9 \%(n=2500 / 28010)$, mientras en el presente estudio es del $41,6 \%$.

Teniendo en cuenta que, según la OMS, la adherencia debe ser superior al 95\% para maximizar los beneficios de la terapia antirretroviral en pacientes con $\mathrm{VIH}+$, y aunque no hay datos disponibles de este tipo para terapia postexposición (PEP), se asume que la magnitud del efecto positivo en cuanto a los resultados con altos niveles de adherencia es similar. ${ }^{4}$ Aunque en este estudio no se puede evaluar la adherencia del usuario al esquema terapéutico, se deduce que los niveles de cumplimiento en la prescripción de la terapia PEP deben ser superiores a los encontrados $(88,6 \%)$, con el fin de minimizar la no adherencia por parte del trabajador, ya que se calcula que un tratamiento profiláctico adecuado disminuye hasta en un $81 \%$ la posibilidad de infección, es decir, la prevención secundaria en este caso no es del 100\%. ${ }^{15}$

No se encontraron datos comparables en la literatura científica indexada, en cuanto al cumplimento de los demás esquemas evaluados, fundamentados básicamente en la realización de los laboratorios sanguíneos, con el fin de medir el riesgo de infección del trabajador expuesto. La adherencia en la adecuada realización de laboratorios para evaluación de riesgo, en cuanto al esquema VIH, fue del 86,1\%; al esquema hepatitis C fue del 83,4\%, registrándose la menor proporción de cumplimiento en la realización de laboratorios, en el esquema para hepatitis $B$, concluyendo una adherencia muy pobre (70\%), teniendo en cuenta que esta patología es considerada como una enfermedad profesional, con un 
Gráfico 5.

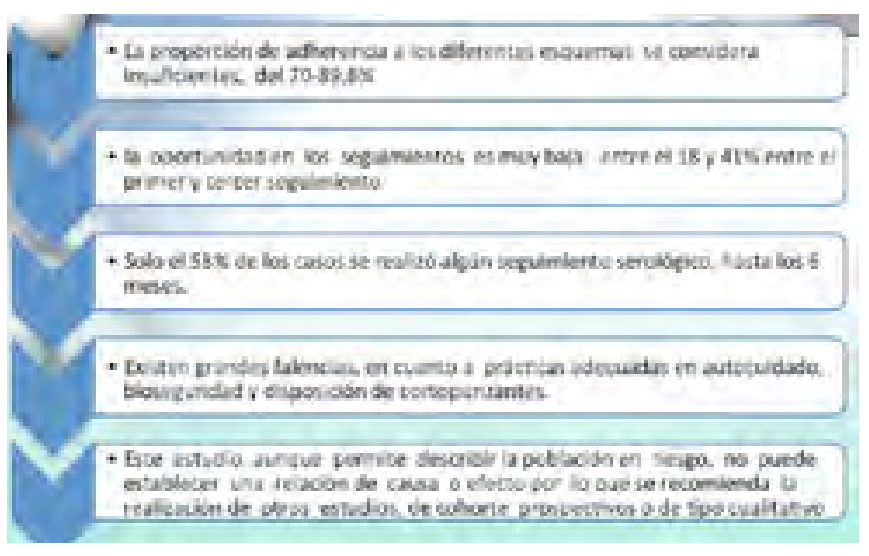

riesgo 10 veces más alto que en la población general ${ }^{13}$ y sumado a que la cobertura de vacunación completa para hepatitis $\mathrm{B}$, en esta población, fue del 67,0\% (995/1485); se considera como preocupante este nivel de cumplimiento en el diagnóstico de riesgo.

Respecto a las posibles causas que pueden influir esta adherencia, varios autores al referirse en general a los diferentes protocolos de algunos eventos en salud, principalmente clínicos no ocupacionales, relacionan como causa de no adherencia el desconocimiento por parte del personal de salud a las guías ${ }^{17}$, hecho que podría también influenciar los presentes resultados, pero que están fuera del alcance del análisis de este estudio. Adicionalmente, se observa una gran diferencia en cuanto a la características del accidente frente a otros países, encontrando que la fuente es desconocida en una gran proporción de los casos -41,6\% (618/1485)-, lo cual hace pensar que en el proceso de atención no hay claridad en la ruta a seguir, cuando el propósito de la actividad o la fuente son desconocidos. En torno al esquema para hepatitis B se debe buscar estrategias para mejorar la adherencia a las guías de manejo ya que se trata del esquema con menores niveles de cumplimiento $-70 \%-$, siendo esta una enfermedad ocupacional claramente definida. ${ }^{14}$ Este estudio, aunque permite describir la población en riesgo, no puede establecer una relación de causa o efecto, por lo que se recomienda la realización de otros estudios, de cohorte prospectivos o de tipo cualitativo, que busquen establecer las causas de la no adherencia a la guías de atención a los accidentes de riesgo biológico.

Respecto a la oportunidad, aunque se observa una oportunidad de menos de 24 horas, en el 88,5\% de los casos se encuentra una inoportunidad muy alta -del 54 al 81\%- en los diferentes, seguimientos serológicos, que deriva en seguimientos prolongados aun después de 6 meses en el 55,3\% de los casos; teniendo en cuenta que se presenta una búsqueda activa en el 98\% de los usuarios (Gráfico 4), lo que nos puede mostrar una conciencia del autocuidado muy baja por parte de los trabajadores afectados.

\section{CONCLUSIONES}

En Colombia el ministerio de Salud, por medio de INS (Instituto Nacional de Salud), y en conjunto con el ministerio de trabajo, deben establecer políticas en salud ocupacional y riesgo laboral, que permitan la iniciación de procesos de vigilancia epidemiológica en la población trabajadora, buscando garantizar su salud, empezando con los que hacen parte de la estructura del sistema sanitario. Es inconcebible que un solo trabajador resulte infectado con alguna de estas enfermedades infecciosas potencialmente mortales, en ejercicio de sus funciones y que adicionalmente tienen una terapéutica tan limitada, existiendo en la actualidad los mecanismos económicos, técnico-científicos y sociales para evitarlo

\section{Agradecimientos}

Dra. Martha Luz Bernal, Médica Especialista en Salud Ocupacional, Gerente Empresa AVENIR SALUD S.A.; Dr. Jorge Fernando Ortiz, Director Nacional Capital Humano Salud y ARL Seguros de Vida Colpatria S.A, Enf. Carmen Luisa Betancur, Directora del Posgrado en Epidemiologia, Fundación Universitaria del Área Andina de Pereira. Gracias a todos por su inmenso apoyo en el transcurso de la investigación.

Trabajo presentado como trabajo de investigación ante jurado académico y director de postgrado de epidemiología de la Fundación Universitaria del Área Andina, Pereira, Risaralda, Colombia, el día 29 de junio de 2013. 


\section{REFERENCIAS}

1. AS, Lane HC. Enfermedad por el Virus de la Inmunodeficiencia Humana SIDA y procesos relacionados. En: Fauci AS. Principios de medicina interna de Harrison. 14 ed. Vol. II. México: McGraw Hill; 1998. p. 2034-2046.

2. Araujo JM, Trujillo-Ferrara JG. De morbisartificum diatriba 1700-2000. Salud Pública Mex.[en línea]2002;44:362-370[consultada nov 2012]. Disponible en: http://www.insp.mx/salud/index.html.

3. Morano LE. Manejo de la exposición ocupacional por VIH y virus de la hepatitis B y C. Rev Panam Infectol. 2004;6(2):43-53.

4. World Health Organization. Guidelines on post-exposure prophylaxis (PEP) to prevent HIV infection. The Joint WHO/ILO Expert Consultation. Geneva: WHO;2005.

5. Prüss-Üstün $A$, Rapiti E, Hutin Y. Sharps injuries: global burden of disease from sharps injuries to health-care workers. World Health Organization; 2003. (WHO Environmental Burden of D Fauci isease Series; 3).

6. Mohr J. Perfil mundial de los trabajadores sanitarios. En: Informe sobre la salud en el mundo Capitulo I. Ginebra: Organización Mundial de la Salud; 2006. p. 3.

7. Cebrián F, Fernández JJ. Riesgo biológico en trabajadores sanitarios, guia práctica para su prevención. Islas Baleares: Govern de les Illes Balears; 2004.

8. Organización Mundial de la Salud. Personal sanitario, infraestructura y medicamentos esenciales. En: Estadisticas Sanitarias Mundiales 2012. Capitulo 6. Ginebra: OMS; 2013.

9. Colombia. Fasecolda. Indicadores técnicos desde el año 2000 al 2011. Bogotá: Fasecolda; 2012.

10. Tolosa EN. Protocolo de vigilancia en salud pública: hepatitis $B, C$ y coinfección hepatitis B-Delta. Bogotá: Instituto Nacional de Salud; 2013.

11. Panlilio A, Cardo D, Campbell $S$, Srivastava P. Estimate of the annual number of percutaneous injuries in U.S. healthcare workers, 4th Decennial
International Conference on Nosocomial and Healthcare Associated Infection, Atlanta, GA, March 5-9, 2000. Atlanta: NaSH Surveillance Group; 2001.

12. USA. Centers for Disease Control and Prevention. Updated U.S. Public Health Service Guidelines for the Management of Occupational Exposures to HBV, $\mathrm{HCV}$, and HIV and Recommendations for Postexposure Prophylaxis. MMWR. 2001;50(RR11):1-42.

13. Centers for Disease Control and Prevention. Updated U.S. Public Health Service guidelines for the management of occupational exposures to HIV and recommendations for Postexposure Prophylaxis. MMWR. 2005; 54(RR-9):1-17.

14. Gutiérrez MN, Sáenz MC. Vacunaciones y profilaxis postexposición en personal sanitario. Departamento de Medicina Preventiva y Salud Pública. Facultad de Medicina. Universidad de Salamanca. Rev Esp Quimioter. 2009; 22(4): 190-200.

15. Cardo DM et al. Case-control study of HIV seroconversion in health-care workers after percutaneous exposure to HIV-infected blood - France, U.K. and U.S., Jan. 1988-Aug 1994. MMWR. 1995; 44: 929-933 or New Engl J Med. 1997, 337:1485-1490.

16. Colombia. Instituto Nacional de Salud. Grupo Enfermedades Transmisibles, Equipo Funcional Infecciones de Transmisión Sexual. Protocolo de vigilancia y control de la hepatitis B. Bogotá: INS; 2011.

17. Machado JE, Isaza B, Sepúlveda M, Ceballos SL. Nivel de adherencia a la guía de atención de neumonia adquirida en la comunidad en el hospital universitario San Jorge de Pereira. Revista Médica de Risaralda. 18(2):134-139.

18. Do AN, Ciesielski CA, Metler RP, Hammett TA, Li J, Fleming PL. Occupationally acquired human immunodeficiency virus (HIV) infection: National case surveillance data during 20 Years of the HIV epidemic in the United States. Infect Cont Hosp Ep. 24(2):86-96. 